\title{
INFLUENCIA DE LOS MÉTODOS DE ENSEÑANZA EN EL APRENDIZAJE Y DESARROLLO DE LA LECTURA
}

\author{
Mª Isabel Marí-Sanmillán \\ Departamento de Ciencias de la Educación \\ Universidad CEU Cardenal Herrera. Castellón de la Plana \\ maria.mari1@uchceu.es \\ Ma Dolores Gil Llario \\ Departamento de Psicología Evolutiva y de la Educación \\ Universidad de Valencia. Estudio General. \\ dolores.gil@uv.es \\ Roberta Ceccato \\ Profesora colaboradora en la Universidad Internacional de Valencia (VIU) \\ roberta.ceccato@campusviu.es
}

Fecha de Recepción: 25 Enero 2019

Fecha de Admisión: 30 Abril 2019

\section{RESUMEN}

En el aprendizaje de la lectura están implicadas habilidades como la conciencia fonológica, la conciencia alfabética o la velocidad de nombramiento. Los distintos métodos de enseñanza influyen de modo diferencial en su adquisición. Este estudio analiza 1) el efecto diferencial de los métodos analíticos y fonológico-sintéticos en la adquisición de estas habilidades básicas, 2) si las diferencias encontradas en función del método siguen el mismo patrón en los 4 primeros cursos de iniciación lectora y 3) la eficacia diferencial de los métodos de enseñanza de la lectoescritura sobre el rendimiento lector en cada curso. Para ello se realizó un estudió transversal. Participaron 614 niños de Educación Infantil (4 y 5 años) y Educación Primaria (6 y 7 años) de colegios de Valencia y Castellón. Los instrumentos aplicados fueron el test RAN (Rapid Automatized Naming) y la batería BIL (de inicio a la lectura) a los 4 y 5 años. Y el test de comprensión lectora ACL, el DST-J (Dyslexia Screening Test) y una subprueba del PROLEC-R a los 6 y 7 años. Los análisis consistieron en unas pruebas $t$ y un MANOVA de dos factores para cada nivel educativo. Los resultados muestran que habilidades como la conciencia fonológica, la velocidad de nombramiento o la conciencia alfabética tienen mayor relevancia durante los primeros años escolares, mientras que la velocidad de nombramiento es la única variable preponderante a los 6 y 7 años. Así mismo, se ha encontrado un mejor rendimiento del método fonológico-sintético en la etapa de Educación Infantil ya que potencia las habilidades con más peso en la fase inicial. Sin embargo, en Educación Primaria es el método global pro- 


\section{INFLUENCIA DE LOS MÉTODOS DE ENSEÑANZA EN EL APRENDIZAJE Y DESARROLLO DE LA LECTURA}

porciona resultados más exitosos en el rendimiento lector. Se confirma así una influencia diferencial de los métodos en cuanto al rendimiento lector dependiendo de la etapa educativa.

Palabras clave: método sintético; método global; RAN; conciencia fonológica; conocimiento alfabético; enseñanza de la lectura

\section{ABSTRACT}

Influences of teaching methods on the learning and development of reading. Learning reading involves skills such as phonological awareness, alphabetic knowledge and naming speed. Different teaching methods have a different effect on their acquisition differently. This study analyses 1) the differential effect of analytic and synthetic phonological methods of acquiring reading skills, 2) whether the differences found, according to the method, followed the same pattern in the first four years of initial reading and 3 ) the effectiveness of the methods of teaching reading on reading performance in each one of the four years of initial reading studies. Therefore, cross-sectional study was conducted. Sample consisted of 614 children of kindergarten (aged 4 and 5), and first and second grade students (aged 6 and 7) from schools located in Valencia and Castellon. Instruments applied was RAN (Rapid Automatized Naming Test) and BIL (Initial Reading Program) (aged 4 and 5), and ACL (Reading Comprehension Test), DST-J (Dyslexia Screening Test) and a subtest of PROLEC-R (Battery for the Evaluation of Reading Processes revised) (aged 6 and 7 ). The analysis consisted of some t-tests to compare the effect of the two study methods at different developmental stages on the analyzed variables and two-factor (year and method) MANOVAs were used for each educational level. Results show the weight of the major reading skills involved vary throughout the process, so phonological awareness, naming speed or alphabetic knowledge have greater relevance during the early school years, while naming speed stands apart as the only variable at ages 6 and 7, when reading requirements change. The synthetic phonological method enhances the most important skills in learning to read in kindergarten, while in Primary Education, the global method provides more successful results in reading performance. The differential influence method has on reading performance also depends on the educational stage.

Keywords: synthetic and analytic methods; RAN; phonological awareness; alphabetic knowledge; teaching reading

\section{INTRODUCCIÓN}

El aprendizaje de la lectura es una de las habilidades más importantes que adquiere el niño durante los primeros años de escolaridad, por este motivo, el modo en cómo enseñar a leer ha sido objeto de numerosos debates (National Reading Panel, 2000). En este sentido, la elección del método de enseñanza, parece importante realizarlo teniendo en cuenta su influencia diferencial sobre el desarrollo de las habilidades cognitivas necesarias para aprender a leer.

En las últimas décadas se han identificado los prerrequisitos, habilidades y factores facilitadores que convierten a un niño en lector competente. Destacan como habilidades predictoras el conocimiento fonológico, el conocimiento alfabético y la velocidad de denominación (Sellés, 2008), y como habilidades facilitadoras el conocimiento metalingüístico acerca del lenguaje escrito y ciertas habilidades lingüísticas cuya base es el desarrollo del lenguaje oral. Esta categoría también integraría procesos cognitivos básicos como la atención, percepción y memoria (Eurydice, 2012). Sólo un adecuado desarrollo de todas ellas permitirá al alumno el acceso a la comprensión.

Tradicionalmente se han distinguido tradicionalmente dos categorías en la enseñanza de la lectura: Ios métodos sintéticos o fonéticos, más centrados en el código, y los métodos analíticos o globales más centrados en el significado. Aunque existen variantes, lo característico de los primeros es 
que comienzan el aprendizaje de las reglas de conversión grafema-fonema al principio del proceso, mientras que los métodos globales lo realizan en etapas más tardías, cuando el niño es capaz de reconocer algunas palabras globalmente (Sellés, 2008). Hasta la fecha, apenas se ha estudiado la influencia de ambos en el desarrollo de los predictores y habilidades facilitadoras de la lectura pero los indicios apuntan a que ambos influyen de manera diferencial en algunas variables cognitivas que intervienen en la lectura (Johnston \& Thompson, 1989; McGeown, Johnston \& Medford, 2012). Según dichos estudios, los métodos sintéticos parecen potenciar habilidades de conciencia fonológica, conocimiento alfabético y memoria verbal a corto plazo, mientras que los métodos analíticos o globales parecen potenciar el desarrollo de vocabulario, conocimiento del alfabeto y memoria visual. Así mismo, la instrucción utilizada en el aprendizaje inicial de lectura parece influir en las estrategias que los niños utilizan en el reconocimiento de palabras y en la lectura (Connelly, Thompson, Fletcher-Flinn \& McKay, 2009), ya que mientras que los métodos sintéticos fomentan el uso de estrategias de decodificación fonológica, los métodos analíticos potencian el desarrollo de estrategias de decodificación ortográfica. No obstante, los estudios no siempre son coincidentes y sobre todo no suelen analizar todas las variables implicadas, ya que se centran en unas u otras. Por ello, se precisan estudios más comprehensivos que analicen la influencia de los métodos de enseñanza de la lectura en la adquisición lectora en niños de E. Infantil y primeros cursos de E. Primaria.

Así pues, los objetivos de este estudio consistieron en primer lugar, en analizar si existe una influencia diferencial según el método de enseñanza de la lectoescritura utilizado en la adquisición y desarrollo de las habilidades básicas implicadas en la capacidad lectora, esto es, velocidad de nombramiento, la conciencia fonológica, el conocimiento alfabético, el conocimiento metalingüístico, las habilidades lingüísticas y los procesos cognitivos. En segundo lugar, analizar si las diferencias encontradas en función del método siguen el mismo patrón en los 4 primeros cursos de iniciación lectora. Y en tercer lugar analizar la eficacia diferencial de los métodos de enseñanza de la lectoescritura sobre el rendimiento lector en cada uno de los 4 cursos de iniciación lectora estudiados.

\section{MÉTODO}

\section{Participantes}

En el estudio participaron 614 niños (265 chicos y 349 chicas) escolarizados en centros educativos ubicados en las provincias de Castellón y Valencia (España). 263 alumnos cursaban $2^{\circ}$ de Educación Infantil (EI) (curso de 4 años), 191 alumnos cursaba $3^{\circ}$ de El (curso de 5 años), 105 cur-

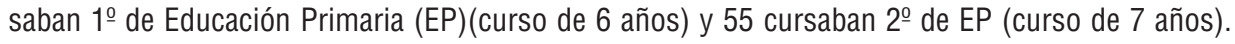
El 52\% estudiaba siguiendo un método fonológico-sintético (Letrilandia)(Usero, 2011) y el 48\% siguiendo un método global basado en la metodología de Glenn Doman (Doman, 2008). Los centros participantes eran de tipo privado-concertado con un nivel socioeconómico similar.

\section{Instrumentos}

Instrumentos aplicados en 4 y 5 años:

Rapid Automatized Naming Test (RAN; Wolf \& Denkla, 2003) consiste en nombrar lo más rápido posible 200 estímulos agrupados en cuatro subtest (números, letras, colores e imágenes familiares). Se anota el tiempo que tarda el alumno en nombrar todos los estímulos de cada cartulina y el número de errores que comete al nombrarlos. Y se obtiene un índice de eficiencia de cada subtest según el procedimiento descrito por Compton (2003) que consiste en convertir las puntuaciones en números/segundo, letras/segundo, colores/segundo e imágenes/segundo. La fiabilidad medida con el alfa de Cronbach es de 0.809 . 


\section{INFLUENCIA DE LOS MÉTODOS DE ENSEÑANZA EN EL APRENDIZAJE Y DESARROLLO DE LA LECTURA}

Batería de Inicio a la Lectura para niños de 3 a 6 años (BIL; Sellés, Martínez, Vidal-Abarca \& Gilabert, 2008) incluye 15 pruebas organizadas en 5 escalas que miden: conciencia fonológica (mediante las subescalas "rimas", "contar palabras", "contar sílabas", "aislar sílabas y fonemas" y "omitir sílabas"), conocimiento alfabético (a través del subtest de "conocimiento del nombre de las letras"), conocimiento metalingüístico (mediante las subescalas "reconocer palabras", "reconocer frases", "funciones de la lectura"), habilidades lingüísticas (a través de los subtest "vocabulario", "articulación", "conceptos básicos" y "estructuras gramaticales") y procesos cognitivos (mediante las subescalas "memoria secuencial auditiva" y "percepción visual”). Además de una medida total (BIL Total) que indica el nivel de madurez para la lectura del niño. En cuanto a la fiabilidad de la prueba, el Alpha de Cronbach de todas pruebas oscila entre 0.54 y 0.97.

Instrumentos aplicados en 6 y 7 años:

RAN (Wolf y Denkla, 2003), anteriormente descrito.

Test de Comprensión Lectora para niños de 1ํa a $6^{\circ}$ de Educación Primaria, ACL (Catalá, Catalá, Molina \& Monclús, 2001). Prueba que evalúa la comprensión lectora a través de cuatro escalas. En este estudio se ha utilizado el primer nivel (ACL-1). La fiabilidad medida con el coeficiente KR-20 es de 0.80 para ACL-1.

Test de detección de la Dislexia en niños, DST-J (Fawcett \& Nicolson, 2004). Es una prueba orientada a niños de 6 a 11 años. Se compone de las escalas "Nombres", "Coordinación", "Lectura de palabras", "Estabilidad postural", "Segmentación fonémica", "Dictado", "Dígitos inversos", "Lectura sin sentido", "Copia", "Fluidez verbal", "Fluidez semántica" y "Vocabulario". La fiabilidad test-retest de la prueba en su adaptación española es de 0.88 .

Batería de Evaluación de los Procesos Lectores revisada, PROLEC-R (Cuetos, Rodríguez, Ruano \& Arribas, 2014) es una batería de evaluación de los procesos lectores para niños de 6 a 12 años. En este estudio se aplicó la subprueba "Nombre de las letras" cuyo objetivo es comprobar si el niño conoce todas las letras y su pronunciación. La fiabilidad del componente "Nombre de las letras" medida con el Alfa de Cronbach es de 0.49 , mientras que la prueba entera presenta una fiabilidad de 0.79 .

\section{Procedimiento}

Tras obtener los permisos oportunos se procedió a la administración de los instrumentos de evaluación. Los 3 evaluadores que participaron en el estudio recibieron una formación específica cuyo objetivo era asegurar que se iban a ofrecer las mismas directrices en la administración de los instrumentos de evaluación. Tras la presentación de cada evaluador a los niños y unos primeros encuentros de familiarización, se administraron los diferentes instrumentos del estudio en sesiones individuales de una duración aproximada de 40 minutos cada una.

\section{RESULTADOS}

Los resultados de las pruebas t mostraron que a los 4 años solo aparecen diferencias a favor de los niños que se iniciaron a la lectoescritura mediante el método fonológico-sintético en las escalas "números" $\left(p=.001^{* *}\right)$ y "colores" $\left(p=.032^{\star}\right)$ de la prueba RAN (tabla 1). Lo que indica una mayor rapidez en el reconocimiento y nombramiento de números y colores con respecto a los alumnos que los que han aprendido a leer con el método global.

A los 5 años, la mayor parte de las habilidades evaluadas muestran medias superiores de forma estadísticamente significativa en los niños que siguen un método fonológico-sintético (tabla 1). Así, se encuentran diferencias significativas en VN: en los componentes "números", "letras" y "colores" $\left(p=.006^{* *} ; p=.014^{*}\right.$ y $p=.006^{* *}$ respectivamente) así como en "Conciencia fonológica" y 
"Conocimiento alfabético" ( $p=.043^{\star}$ y $\left.p<.001^{* *}\right)$. Así mismo, se ha podido constatar un nivel significativamente superior en la puntuación total de la BIL lo que muestra que los niños que aprenden mediante un método sintético poseen una mayor madurez lectora que los que aprenden mediante un método global (tabla 1).

A los 6 años (tabla 2) sólo hay diferencias significativas en el componente de "colores" de la RAN $\left(p=.043^{*}\right)$, a favor del método fonológico-sintético. Mientras que el método global se muestra superior en "Fluidez Verbal" ( $\left.p=.050^{\star}\right)$, "Fluidez Semántica" $\left(p=.019^{\star}\right)$, rendimiento lector, ya que las medias en "Nombre de las letras" $\left(p<.001^{\star *}\right)$, "Lectura de palabras" $\left(p=.031^{\star}\right)$ y "Dictado" $\left(p<.001^{* *}\right)$ son significativamente superiores en este grupo, y por último en todas las variables de comprensión lectora ("Comprensión literal”, $p=.005^{\star *}$; "Reorganización”, $p=.021^{\star}$; "Comprensión crítica", $p=.003^{* *}$; “Comprensión Lectora Total", $p=.005^{* *}$ y puntuación media de la prueba "ACL media", $\left.p=.005^{\star *}\right)$.

Los resultados hallados a los 7 años muestran, por el contrario, que los niños que han aprendido con el método global obtienen un rendimiento superior en las medias de "nombre de las letras" $\left(p=.003^{* *}\right)$, "Lectura de palabras" $\left(p=.01^{* *}\right)$ y "Dictado" $\left(p<.001^{* *}\right)$. Así mismo, se confirma la fuerza del método sintético en el componente "Colores" de la RAN $\left(p=.026^{\star}\right)$ (tabla 2$)$.

Tabla 1

Comparación entre métodos de enseñanza de la lectoescritura en 4 y 5 años

\begin{tabular}{|c|c|c|c|c|c|c|}
\hline & \multicolumn{3}{|c|}{4 Aก̃ $0 \mathrm{~S}$} & \multicolumn{3}{|c|}{5 AÑOS } \\
\hline & $\begin{array}{c}\text { Método global } \\
\bar{X}(\mathrm{DT})\end{array}$ & $\begin{array}{c}\text { Método sintético } \\
\bar{X}(\mathrm{DT})\end{array}$ & $\mathrm{T}(\mathrm{p})$ & $\begin{array}{c}\text { Método global } \\
\bar{X} \text { (DT) }\end{array}$ & $\begin{array}{c}\text { Método sintético } \\
\bar{X}(\mathrm{DT})\end{array}$ & $T(p)$ \\
\hline RAN números & $.743(.557)$ & $1.015(.597)$ & $-3.296\left(.001^{* *}\right)$ & $1.022(.373)$ & $1.169(.339)$ & $-2.805\left(.006^{* *}\right)$ \\
\hline RAN letras & $.674(.374)$ & $.772(.413)$ & $-1.584(.115)$ & $1.009(.355)$ & $1.138(.348)$ & $-2.49\left(.014^{*}\right)$ \\
\hline RAN colores & $.995(.676)$ & $1.181(.692)$ & $-2.155\left(.032^{\circ}\right)$ & $.727(.208)$ & $.816(.228)$ & $-2.76\left(.006^{* * *}\right)$ \\
\hline RAN imágenes & $1.058(.686)$ & $1.225(.717)$ & $-1.898(.059)$ & $.734(.181)$ & $.785(.196)$ & $-1.824(.07)$ \\
\hline Conciencia fonológica & $16.939(6.548)$ & $17.966(.805)$ & $-1.14(.255)$ & $27.107(7.358)$ & $29.280(7.124)$ & $-2.034\left(.043^{*}\right)$ \\
\hline $\begin{array}{l}\text { Conocimiento } \\
\text { alfabético }\end{array}$ & $12.08(6.289)$ & $12.28(7.417)$ & $-.235(.814)$ & $21.05(3.354)$ & $22.60(2.149)$ & $-3.742(<.001 * * *)$ \\
\hline $\begin{array}{l}\text { Conocimiento } \\
\text { metalingüístico }\end{array}$ & $10.574(2.968)$ & $10.733(3.22)$ & $-.411(.681)$ & $13.328(1.649)$ & $13.693(1.471)$ & $-1.580(.116)$ \\
\hline $\begin{array}{l}\text { Habilidades } \\
\text { Lingüísticas }\end{array}$ & $22.748(4.147)$ & $22.875(4.406)$ & $-.238(.812)$ & $26.247(2.556)$ & $26.203(3.136)$ & $.104(.917)$ \\
\hline Procesos cognitivos & $31.655(6.984)$ & $31.973(8.123)$ & $-.334(.738)$ & $39.462(4.438)$ & $40.399(4.6178)$ & $-1.403(.162)$ \\
\hline BIL total & $59.963(14.521)$ & $61.458(17.958)$ & $-746(.456)$ & $83.804(10.953)$ & $87.658(10.895)$ & $-2.393\left(.018^{*}\right)$ \\
\hline
\end{tabular}

Nota: ${ }^{*} p<.05,{ }^{* *} p<.001$ 
Tabla 2

Comparación entre métodos de enseñanza de la lectoescritura en 6 y 7 años

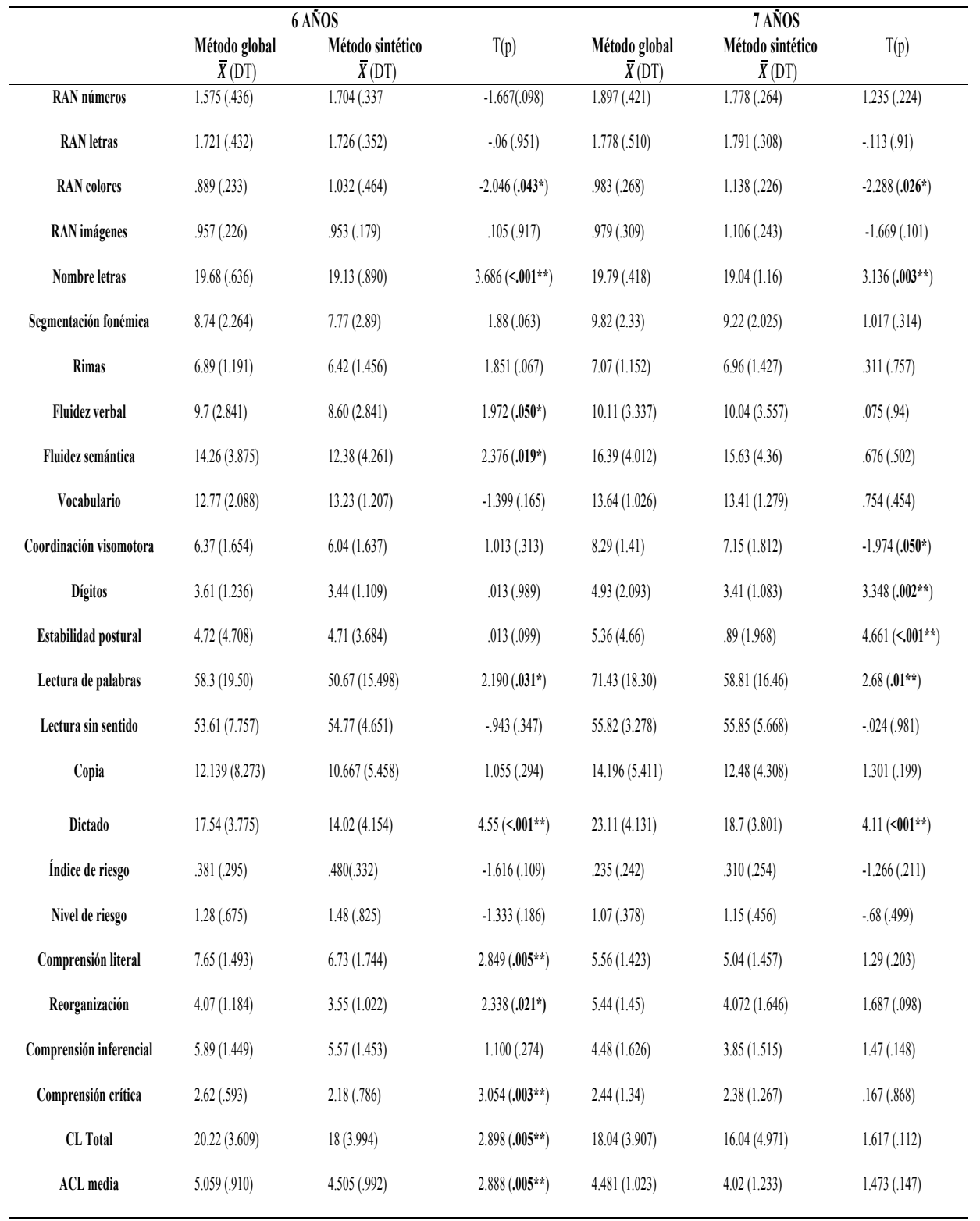

Nota: ${ }^{*} p<.05 ;{ }^{* *} p<.001$ 
Nuestro segundo objetivo fue analizar si las diferencias encontradas entre los grupos se deben al método o sólo al curso. El MANOVA relativo a los cursos de El muestra un efecto del factor "curso" en todas las variables analizadas (ver tabla 3). Así mismo, muestra un efecto significativo del factor "método" en los componentes de "Conciencia fonológica" y "BIL Total" en los que obtienen mejores resultados los niños que aprenden a leer a través del método fonológico-sintético. Sin embargo, no se encontró interacción de los dos factores "curso*método" en ninguna de las variables analizadas en 4 y 5 años.

Tabla 3. MANOVA de los factores curso y método en E. Infantil.

\begin{tabular}{|c|c|c|c|c|c|c|c|c|c|}
\hline & \multicolumn{3}{|c|}{ Curso } & \multicolumn{3}{|c|}{ Método } & \multicolumn{3}{|c|}{ Curso*Método } \\
\hline & $\mathbf{F}$ & Sig & gl & $\mathbf{F}$ & Sig & gl & $\mathbf{F}$ & Sig. & gl \\
\hline $\begin{array}{l}\text { Conciencia } \\
\text { fonológica }\end{array}$ & 231.166 & $<.001 * *$ & 1 & 5.328 & $.021 *$ & 1 & .573 & .450 & 1 \\
\hline $\begin{array}{l}\text { Conocimiento } \\
\text { alfabético }\end{array}$ & 318.157 & $<.001 * *$ & 1 & 2.733 & .099 & 1 & 1.454 & .228 & 1 \\
\hline $\begin{array}{l}\text { Conocimiento } \\
\text { metalingüístico }\end{array}$ & 132.134 & $<.001 * *$ & 1 & 1.301 & .255 & 1 & .098 & .754 & 1 \\
\hline $\begin{array}{l}\text { Habilidades } \\
\text { lingüísticas }\end{array}$ & 89.247 & $<.001 * *$ & 1 & .029 & .865 & 1 & .085 & .771 & 1 \\
\hline $\begin{array}{l}\text { Procesos } \\
\text { Cognitivos }\end{array}$ & 166.721 & $<.001 * *$ & 1 & 1.112 & .292 & 1 & .184 & .668 & 1 \\
\hline BIL Total & 322.212 & $<.001 * *$ & 1 & 3.930 & $.048 *$ & 1 & .596 & .441 & 1 \\
\hline
\end{tabular}

Nota: ${ }^{*} p<.05 ;^{* *} p<.001$

EI MANOVA de la etapa de E. Primaria muestra un efecto significativo del factor "método" en los componentes de "Dígitos" $\left(p<.001^{* \star}\right)$ "Estabilidad postural" $\left(p=.001^{* *}\right)$, "Lectura de palabras" $\left(p=.001^{* *}\right)$, "Dictado" $\left(p<.001^{* *}\right)$, “Comprensión literal" $\left(p=.008^{* *}\right)$, "Reorganización" $\left(p=.005^{* *}\right)$, Comprensión Lectora Total $\left(p=.003^{* *}\right)$ y media de toda la prueba (ACL media) $\left(p=.004^{\star *}\right)$. LoS niños que aprenden mediante un método de lecto-escritura global, obtienen puntuaciones mejores. También se observa un efecto significativo de la interacción entre los dos factores (curso*método) en las variables de "Coordinación visomotora" $\left(p=.024^{*}\right)$, "Dígitos" $\left(p=.004^{* *}\right)$ y "Estabilidad postural" $\left(p=.001^{* \star}\right)$, por lo que en estas variables las puntuaciones obtenidas por los niños en los diferentes momentos de desarrollo variarían de forma significativa en función del método utilizado.

Por último, los resultados de la clusterización (para seleccionar a los niños de alto y bajo rendimiento, con percentil superior a 75 e inferior a 25 respectivamente) mostraron que a los 4 años no hay diferencias entre métodos (tabla 4); a los 5 años existe una diferencia significativa entre métodos $\left(p=.004^{* *}\right)$ asociándose el mejor rendimiento al método sintético y el peor rendimiento al método analítico; a los 6 años se observa una diferencia significativa entre métodos $\left(p=.028^{*}\right)$, mostrando un mejor rendimiento el método global y un peor rendimiento el método sintético. Por último, a los 7 años no existen diferencias significativas entre los dos métodos distribuyéndose de forma bastante homogénea tanto en bajo como en alto rendimiento $(p=.431)$. 


\section{INFLUENCIA DE LOS MÉTODOS DE ENSEÑANZA EN EL APRENDIZAJE Y DESARROLLO DE LA LECTURA}

Tabla 4. Distribución de los alumnos de alto/bajo rendimiento en los dos métodos de enseñanza de la lectoescritura y chi cuadrado de comparación en 4, 5, 6 y 7 años.

\begin{tabular}{ccccccc}
\hline Edad & Rendimiento & $\begin{array}{c}\text { Número } \\
\text { alumnos }\end{array}$ & $\begin{array}{c}\text { Método } \\
\text { global }\end{array}$ & $\begin{array}{c}\text { Método } \\
\text { fonológico- } \\
\text { sintético }\end{array}$ & $\begin{array}{c}\text { Chi- } \\
\text { cuadrado }\end{array}$ & p \\
\hline $\mathbf{4}$ años & Bajo & 64 & $25(21 \%)$ & $39(26 \%)$ & .976 & .323 \\
& Alto & 60 & $20(17 \%)$ & $40(27 \%)$ & & \\
$\mathbf{5}$ años & Bajo & 46 & $30(30 \%)$ & $16(17 \%)$ & 8.522 &. $\mathbf{0 0 4 * *}$ \\
& Alto & 46 & $16(16 \%)$ & $30(31 \%)$ & & \\
$\mathbf{6}$ años & Bajo & 27 & $10(18 \%)$ & $17(32 \%)$ & 4.800 & $\mathbf{. 0 2 8 *}$ \\
& Alto & 32 & $21(39 \%)$ & $11(21 \%)$ & & \\
$\mathbf{7}$ años & Bajo & 13 & $5(17 \%)$ & $8(29 \%)$ & .619 & .431 \\
& Alto & 13 & $7(25 \%)$ & $6(20 \%)$ & & \\
\hline
\end{tabular}

Nota: los porcentajes están calculados en base a la totalidad de niños participantes en cada método. Nota: $* \mathrm{p}<.05 ; * * \mathrm{p}<.001$

\section{DISCUSIÓN}

Los resultados obtenidos en cuanto a la capacidad diferencial de los métodos para potenciar el desarrollo de las habilidades implicadas en la adquisición de la lectura muestran que los niños iniciados con el método fonológico-sintético desarrollan más tempranamente y con mayor dominio la $C F$, el $C A$ y la VN. Sin embargo, a partir de los 6 años, una vez el niño domina las reglas de conversión grafema-fonema (RCGF) y los procesos de decodificación, la influencia de esas variables tiende a desaparecer y con ella la del método con el que se inició su aprendizaje. La explicación apunta a que dicho método incide directamente en la adquisición de las RCGF (Cuetos, 2010; McGeown et al., 2012), y confirmaría, en la línea de Onochie-Quintanilla, Simpson, Caravolas y Defior (2011), que la adquisición del control automático del procedimiento fonológico en lenguas transparentes tiene lugar durante las etapas iniciales del aprendizaje de la lectoescritura, cuando la adquisición del código aún no es completa. Por otro lado, el hecho de que el método fonológico-sintético fomente la adquisición temprana de la VN podría deberse a que la implicación de la VN en el acceso y recuperación de los códigos fonológicos de la memoria a largo plazo, aspecto trabajado mediante este método y no tanto el acceso a los procesos de naturaleza ortográfica., apoyando, por tanto, la concepción de la VN como un aspecto del procesamiento fonológico (Araújo et al, 2011; Georgiou, Parrila, Kirby \& Stephenson, 2008). Así mismo, parece comprensible que la VN aparezca antes que la $C F$, en cuanto que la $V N$ es una habilidad fonológica implícita en la que los códigos fonológicos se recuperan automáticamente, sin necesidad de reflexión explícita sobre ellos (Defior \& Serrano, 2011).

Respecto al método asociado con un mejor rendimiento lector, los resultados indican que en Educación Infantil es el método sintético. Sin embargo, en edades posteriores cuando el niño empieza a dominar la mecánica de la lectura y el alumno incorpora estrategias lectoras diferentes, encontramos que el método que potencia la $V N$ y que proporciona resultados más exitosos en el rendimiento lector es el método global. Esto confirmaría que la adquisición y desarrollo de la habilidad lectora implica un cambio de estrategias en la lectura que va de la decodificación lenta y secuencial hasta el reconocimiento rápido de la palabra completa (Araujo et al., 2011). El método global parece favorecer el desarrollo de la fluidez y la precisión gracias a su capacidad de desarrollo de las 
estrategias de tipo ortográfico (López-Escribano \& Katzir, 2008). Así mismo, estos resultados confirman la tesis propuesta por Carril (2008) quien afirma que el lector eficaz tiende a la lectura global, ya que ésta es un facilitador de la comprensión y la velocidad lectora.

En conclusión, podemos señalar que las habilidades y estrategias utilizadas en las distintas fases de la adquisición lectora van variando en función de los estadios que atraviesa el alumno. De tal modo que habilidades precursoras como la $C F$, la $V N$ y el $C A$ presentan una mayor relevancia durante los primeros años para dejar paso a la preponderancia única de la $V N$ a partir del momento en el que cambian los requerimientos de la lectura, cuando el alumno pasa de centrar sus recursos cognitivos en la tarea de decodificación a una decodificación automática y fluida de las palabras que propicia la comprensión. Puesto que cada método se ha mostrado diferencialmente idóneo para la consecución de estos dos objetivos podrían resultar diferencialmente eficaces en los distintos momentos del aprendizaje lector, cuestión que convendrá tener en cuenta por el profesorado para incorporar estrategias de enseñanza de lectura más de tipo sintético en el inicio de Educación Infantil y de tipo global en los cursos posteriores.

\section{REFERENCIAS BIBLIOGRÁFICAS}

Araújo, S., Inácio, F., Francisco, A., Faísca, L., Petersson, K. M., \& Reis, A. (2011). Component processes subserving rapid automatized naming in dyslexic and non-dyslexic readers. Dyslexia, 17, 242-255.

Carril, I., (2008, febrero). Pasado, presente y futuro de la metodología. Ponencia presentada en el Congreso Internacional de animación a la lectura. Morelia-Michoacán-México. Recuperado 5 enero, 2015 de http://www.waece.org/memoriascongresos/morelia2008/PDF/PONENCIA01.pdf

Català, G., Català, M.; Molina, E., \& Monclús, R. (2001). Evaluación de la Comprensión Lectora. Pruebas ACL (1-6 grado de primaria). España: Editorial Graó.

Connelly, V., Thompson, G. B., Fletcher-Flinn, C. M., \& McKay, M. F. (2009). Does the type of reading instruction have an influence on how readers process print? In C. Wood, \& V. Connelly (Eds.), Contemporary perspectives in reading and spelling (pp. 239-253). London: Routledge.

Georgiou, G. K., Parrila, R., Kirby, J. R., \& Stephenson, K. (2008). Rapid naming components and their relationship with phonological awareness, orthographic knowledge, speed of processing, and different reading outcomes. Scientific Studies of Reading, 12, 325-350.

Cuetos, F. (2010). Psicología de la lectura. Madrid: Wolters Kluwer España.

Cuetos, F., Rodríguez, B., Ruano, E., \& Arribas, D. (2014). PROLEC-R. Batería de evaluación de los procesos lectores, revisada (5⿳亠丷厂 edición). Madrid: TEA Ediciones.

Defior, S. \& Serrano, F. (2011). Procesos fonológicos explícitos e implícitos. Lectura y Dislexia. Revista de Neuropsicología, Neuropsiquiatría y Neurociencias, 11, 79-94.

Eurydice. Ministerio de Educación, Cultura y Deporte (2012). La atención al alumnado con dislexia en el sistema educativo en el contexto de las necesidades específicas de apoyo educativo. España: Colección Eurydice España-Redie. Recuperado 6 febrero, 2016 de https://sede.educacion.gob.es/publiventa/la-atencion-al-alumnado-con-dislexia-en-el-sistema-educativo-en-elcontexto-de-las-necesidades-especificas-de-apoyo-educativo/educacion-especial-y-compensatoria/15956

Fawcett, A.J. \& Nicolson, R.I. (2004). Test para la Detección de la Dislexia en Niños, DST-J. Oxford. Pearson Assesment. Adaptación española (2010) Madrid. TEA

Johnston, R. S., \& Thompson, G. B. (1989). Is dependence on phonological information in children's reading a product of instructional approach? Journal of Experimental Child Psychology, 48, 131-145. 


\section{INFLUENCIA DE LOS MÉTODOS DE ENSEÑANZA EN EL APRENDIZAJE Y DESARROLLO DE LA LECTURA}

López-Escribano, C. \& Katzir, T. (2008). Are phonological processes separate from the processes underlying naming speed in a shallow orthography? Education Psychology, 16, 641-666.

McGeown, S. P., Johnston, R. S., \& Medford, E. (2012). Reading instruction affects the cognitive skills supporting early reading development. Learning and Individual Differences, 22(3), 360364.

National Institute of Child Health and Human Development (2000). Report of the National Reading Panel: Teaching children to read: An evidence-based assessment of the scientific research literature on reading and its implications for reading instruction. Reports of the subgroups (NIH Publication No. 00-4769). Washington, DC: U.S. Government Printing Office.

Onochie-Quintanilla, E., Simpson, I, Caravolas, M. \& Defior, S. (2011). Letter knowledge, phoneme awareness and RAN as predictors of reading fluency in Spanish. 10th Symposium of Psycholinguistics, San Sebastián, April 13th-16 th

Sellés, P. (2008). Elaboración de una prueba de habilidades relacionadas con el desarrollo inicial de la lectura (BIL 3-6). Tesis. Universitat de Valencia, Valencia.

Sellés, P., Martínez, T., Vidal-Abarca, E. \& Gilabert, R. (2008). Batería de inicio a la lectura para niños de 3 a 6 años. Madrid: ICCE.

Usero Alijarde, A. (2011). Letrilandia. Libros de lectura. Propuesta didáctica. Zaragoza: Edelvives. Wolf, M., \& Denckla, M. (2003). Rapid Automatized Naming Tests. Greenville, SC: Super Duper. 